\title{
Generalized Cav(u) Theorem for Repeated Games with One-sided Incomplete Information
}

\author{
Weicheng Xu, ${ }^{1,2^{*}}$ Zhendong Zhang, ${ }^{1}$ Xiaoyi Feng ${ }^{1}$ \\ ${ }^{1}$ School of Economics, Ocean University of China, Qingdao 266100, China. \\ ${ }^{2}$ Institute of Marine Development, Ocean University of China, Qingdao 266100, China. \\ * Author to whom correspondence should be addressed; E-Mail: ouctgyx@126.com
}

\begin{abstract}
This study was devoted to extend $\operatorname{Cav}(\mathrm{u})$ theorem for repeated games with one-sided incomplete information. The model considered in this paper was based on the typical Aumann-Maschler model. According to the properties of Wasserstein distance, the Cav(u) theorem for repeated games with one-sided incomplete information was generalized from convex and compact subset to the whole space. And an approximation method was provided for dealing with the problem of probability measurement in the whole space.
\end{abstract}

Keywords: One-sided incomplete information; Repeated game; $\mathrm{Cav}(\mathrm{u})$ theorem; Wasserstein distance 


\section{Introduction}

The Aumann-Maschler model [1] is a typical repeated game model with one-sided incomplete information, which assumes that one of the two sides of the game can obtain the exact value of the state variable in advance while the other party only knows the probability distribution of the state variable (One-sided incomplete information). The state set and strategy set of both parties of the game are finite sets; the game is repeated and the strategies of both parties are disclosed after each round. In each round, the two sides of the game choose the best strategy according to the information they know. It is a zero-sum game. De Meyer B, Lehrer E, Rosenberg D [2] studied zero-sum games with incomplete information and identified the Blackwell monotonicity. Renault, Jérôme [3] generalized the proof of Aumann and Maschler, and, proved the existence of the uniform value from the definition and the study of appropriate nonrevealing auxiliary games with infinitely many stages. Fabien Gensbittel, Jérôme Renault [4] showed a more involved model that the model of two-player zero-sum repeated game with lack of information on both sides and perfect observation, which revealed that the infinitely repeated game may have no value. Under these assumptions, the Cav(u) theorem was obtained in [1], that is, the average value of the one-sided incomplete information game with the increase of rounds approaches the concave generalization of the value of single-round complete information games, meanings that as game rounds increases, the party who has internal information in advance will gradually lose the information advantage. Neyman and Abraham [5] proved that for two persons repeated game, it can be simplified as a typical repeated game with one-sided incomplete information under some conditions, which can help us better understand the incomplete information game. By another proof of Aumann and Maschler's theorem, Bernard De Meyer and Dinah Rosenberg [6] characterized that the limit value of a finite repeated game without information is the concave of the value of a game without information.

On the basis of [1], De Meyer [7] extended the repeated game model to the situation that both the state set and strategy set are both infinite sets, proposed the concept of continuous maximum variation martingale, and gave this kind of game model in finance in the application. Gensbittel [8][9] extended the repeated game model in [7] from one-dimensional 
case to multi-dimensional case. In [8] and [9], we obtained the $\mathrm{Cav}(\mathrm{u})$ theorem when the state variable is defined in a finite set and a convex compact subset defined in $\boldsymbol{R}^{d}$, respectively. Laraki R [10] used the recursive structure for the finitely repeated version of dual games, to identify the limit value of a repeated game in discrete time. On the application of Wasserstein distance, Pedro C. Alvarez-Esteban [11] applied the property of Wasserstein distance to the trimming methodology, forming a robust method to evaluate the suitability of data samples, which can be considered to be correct, giving us an idea to introduce Wasserstein distance into the proof of the theorem.

In the latest game study, Juan F. Escobar and Gastón Llanes [12] also analyzed cooperation dynamics in repeated games with Markovian private information, showing that the way in which private information is transmitted may be aggressive, and partners will allow a certain number of ways for transmitting information. We also proved that when the interest rate tends to be zero, the welfare loss will gradually disappear as the type process continues to increase. Ashkenazi-Golan, G; Rainer, C and Solan, E [13] explored the optimal use of information in Markov games with one-sided incomplete information and two states. A finite stage algorithm was proposed to calculate the limit value when the interval becomes zero, and the optimal strategy in the continuous time limited game was given. Ueda, Masahiko [14] put forward the modified zero decisive strategies, and the prisoner's dilemma of repeated game as one of the strategies, to balance the probability distribution functions of payoffs.

This paper also considered a more general situation. Assuming that the state variables in the repeated game were defined in the entire space $\boldsymbol{R}^{d}$, at this time, the corresponding $\operatorname{Cav}(\mathrm{u})$ theorem was proved with the properties of Wasserstein distance.

\section{Repeated game model with one-sided incomplete information}

Considering the two-man zero-sum repeated game model with one-sided incomplete information:

The strategy sets of both participants are $I$ and $J$ (not necessarily a finite set), and the Borel- $\sigma$ on them are I and $\vartheta$ respectively. $T$ is a bounded Borel mapping from $I \times J$ to $\boldsymbol{R}^{d}$. The state 
variable is a vector $L=\left(L^{1}, \Lambda, L^{d}\right) \in \boldsymbol{R}^{d}$. Given the probability measure $\mu \in \Delta^{p}\left(\boldsymbol{R}^{d}\right)$ on $\boldsymbol{R}^{d}$ where $\Delta^{p}\left(\boldsymbol{R}^{d}\right)$ represents the set of probability measures with finite moments of $p$ on $\boldsymbol{R}^{d}$, the repeated game in Round $n-$ is conducted according to the following rules:

Round 0: Choose $L$ with probability measure $\mu$ beforehand. Participant 1 knows the exact value of the random variable $L$, which Participant 2 only knows that the probability distribution of $L$ is $\mu$.

Round q $(q=1, \Lambda, n)$ : Both Participant 1 and Participant 2 adopt their own strategies $i_{q} \in I$ and $j_{q} \in J$ independently according to their own information and historical observation. And then, they will disclose their strategy to each other after each round. Formally, the behavior strategy of Participant $1 \sigma$ is a list of transition probabilities depending on his internal information (the exact value of $L$ ) and historical observations $\left(\sigma_{1}, \Lambda, \sigma_{n}\right)$ :

$$
\sigma_{q}: \boldsymbol{R}^{d} \times(I \times J)^{q-1} \rightarrow \Delta(I)
$$

Among them, $\sigma_{q}\left(L, i_{1}, j_{1}, \Lambda, i_{q-1}, j_{q-1}\right)$ means that the qth round, when the value of the state variable is $L$, and the historical strategy for the first $q-1$ round of the game is $\left(i_{1}, j_{1}, \Lambda, i_{q-1}, j_{q-1}\right)$, Participant 1 will adopt the probability distribution of strategy $i_{q}$. The symbol $\Delta(I)$ represents the totality of probability measures on $I$. Similarly, Participant 2's behavior strategy $\tau$ is a list of transition probabilities depending on his historical observations $\left(\tau_{1}, \Lambda, \tau_{n}\right):$

$$
\tau_{q}:(I \times J)^{q-1} \rightarrow \Delta(J) 。
$$

Denote the behavioral strategy sets of Participant 1 and Participant 2 as $\Sigma_{n}$ and $\mathrm{T}_{n}$, respectively. Comparing formula (1) and formula (2), we can find that the strategy of Participant 1 depends on the state variable $L$ and the strategy of Participant 2 is independent of the state variable $L$, thus the game is a one-sided incomplete information game. According to Tulcea theorem, the combination of ternary variables $(\mu, \sigma, \tau)$ can induce a unique probability 
measure $\pi(\mu, \sigma, \tau) \in \Delta\left(\boldsymbol{R}^{d} \times I^{n} \times J^{n}\right)$. And in this paper, only the zero-sum game is considered, and the payout function of Participant 1 is defined as:

$$
g_{n}(\mu, \sigma, \tau)=E_{\pi(\mu, \sigma, \tau)}\left[\left\langle L, \sum_{q=1}^{n} T\left(i_{q}, j_{q}\right)\right\rangle\right]
$$

Assuming that all participants seek to maximize their own income, the maximum payment of Participant 1 and the minimum payment of participant 2 are respectively:

$$
\begin{aligned}
& \underline{V}_{n}(\mu)=\operatorname{supinf}_{\sigma \in \Sigma_{n} \tau \in \mathrm{T}_{n}} E_{\pi(\mu, \sigma, \tau)}\left[\left\langle L, \sum_{q=1}^{n} T\left(i_{q}, j_{q}\right)\right\rangle\right], \\
& \bar{V}_{n}(\mu)=\inf _{\tau \in \mathrm{T}_{n} \sigma \in \Sigma_{n}} \sup _{\pi(\mu, \sigma, \tau)}\left[\left\langle L, \sum_{q=1}^{n} T\left(i_{q}, j_{q}\right)\right\rangle\right] 。
\end{aligned}
$$

Once the above game rules are given, the final result of the game depends only on the probability distribution $\mu$ of state variables $L$ and game rounds $n$, therefore, this paper will abbreviate this kind of game as $\Gamma_{n}(\mu)$.

Theorem 1.1 Assuming $p \geq 1$, Functions $\underline{V}_{n}$ and $\bar{V}_{n}$ will satisfy the following properties:

(i) Concavity: $\underline{V}_{n}$ and $\bar{V}_{n}$ are concave functions on $\Delta^{p}\left(\boldsymbol{R}^{d}\right)$

(ii) Lipschitz continuity:

$$
\left|\frac{1}{n} \underline{V}_{n}(\mu)-\frac{1}{n} \underline{V}_{n}(v)\right| \leq K W_{p}(\mu, v) ;\left|\frac{1}{n} \bar{V}_{n}(\mu)-\frac{1}{n} \bar{V}_{n}(v)\right| \leq K W_{p}(\mu, v)
$$

Where $K$ is the upper bound of Borel mapping $T$.

(iii) Homogeneity:

$$
\forall \lambda>0, \underline{V}_{n}([\lambda X])=\lambda \underline{V}_{n}([X]), \bar{V}_{n}([\lambda X])=\lambda \bar{V}_{n}([X])
$$

In theorem $1.1,[X]$ represents the probability distribution of random variable $X, W_{p}(\mu, v)$ represents the Wasserstein distance of order $p$, which is defined as:

$$
W_{p}(\mu, v)=\inf \left\{E\left[|X-Y|^{p}\right]^{\frac{1}{p}},[X]=\mu,[Y]=v\right\}
$$

Unless otherwise specified in this paper, all is assumed to be $p \geq 1$

Proof: According to Formula (4) and (5), positive homogeneity is typical. Concavity and 
Lipschitz continuity are proved as follows, taking $\underline{V}_{n}$ as an example:

(i) Suppose $\mu_{1}, \mu_{2} \in \Delta^{p}\left(\boldsymbol{R}^{d}\right)$ and $\mu=\lambda \mu_{1}+(1-\lambda) \mu_{2}$, where $\lambda \in(0,1)$. Consider that Participant 1 takes the following strategy in $\Gamma_{n}(\mu)$ : takes the $\varepsilon-$ optimal strategy in $\Gamma_{n}\left(\mu_{1}\right)$ with the probability of $\lambda$, takes the $\varepsilon$ - optimal strategy in $\Gamma_{n}\left(\mu_{2}\right)$ with the probability of $1-\lambda$. Under this hybrid strategy, the final return of Participant 1 is $\lambda \underline{V}_{n}\left(\mu_{1}\right)+(1-\lambda) \underline{V}_{n}\left(\mu_{2}\right)-\varepsilon$, since $\varepsilon$ can be arbitrarily small, then $\underline{V}_{n}(\mu) \geq \lambda \underline{V}_{n}\left(\mu_{1}\right)+(1-\lambda) \underline{V}_{n}\left(\mu_{2}\right)$, we can see that $\underline{V}_{n}$ is a concave function.

(ii) Suppose $\left[L_{1}\right]=\mu,\left[L_{2}\right]=v$, then:

$$
\begin{aligned}
& \mid E_{\pi(\mu, \sigma, \tau)}\left[\left\langle L_{1}, \sum_{q=1}^{n} T\left(i_{q}, j_{q}\right)\right|\right]-E_{\pi(\mu, \sigma, \tau)}\left\lfloor\left\langle L_{2}, \sum_{q=1}^{n} T\left(i_{q}, j_{q}\right)\right\rangle||\right. \\
& \leq E_{\pi(\mu, \sigma, \tau)}\left[\left|\left\langle L_{1}-L_{2}, \sum_{q=1}^{n} T\left(i_{q}, j_{q}\right)\right\rangle\right|\right] \leq n K W_{1}(\mu, v)
\end{aligned}
$$

So, we can get $\left|\frac{1}{n} \underline{V}_{n}(\mu)-\frac{1}{n} \underline{V}_{n}(v)\right| \leq K W_{1}(\mu, v) \leq K W_{p}(\mu, v)$. The same can be proved for $\bar{V}_{n}$

After comparing Equation (4) and (5), it is easy to see that $\underline{V}_{n}(\mu) \leq \bar{V}_{n}(\mu)$. Different from the typical Aumann-Maschler model, because $I$ and $J$ are not necessarily finite sets, the minimax theorem may not always be held. If they are equal, then the value of game $\Gamma_{n}(\mu)$ exists, which is denoted as:

$$
V_{n}(\mu):=\underline{V}_{n}(\mu)=\bar{V}_{n}(\mu)
$$

As a special case of repeated game with one-sided incomplete information, the single-round complete information game is considered. In this case, participant 1 does not know the exact state of $L$, so the $L-$ independent policy set of participant 1 can be regarded as $\Delta(I)$. Similarly, the maximum payment of Participant 1 and the minimum payment of Participant 2 are respectively defined as:

$$
\underline{u}(\mu):=\sup _{\sigma \in \Delta(I) \tau \in \Delta(J)} \inf _{\pi(\mu, \sigma, \tau)}\left[\left\langle L, T\left(i_{q}, j_{q}\right)\right\rangle\right],
$$




$$
\bar{u}(\mu):=\inf _{\tau \in \Delta(J) \sigma \in \Delta(I)} \sup _{\pi(\mu, \sigma, \tau)}\left[\left\langle L, T\left(i_{q}, j_{q}\right)\right\rangle\right]
$$

Particularly, it is easy to get the following results:

Theorem 1.2 For all $\mu \in \Delta^{p}\left(\boldsymbol{R}^{d}\right)$, there are:

$$
\begin{aligned}
& \underline{u}(\mu)=\underline{V}_{1}([E(\mu)]), \\
& \bar{u}(\mu)=\bar{V}_{1}([E(\mu)]),
\end{aligned}
$$

where, $E(\mu)$ represents the expected value of probability measure $\mu$.

Functions $\underline{u}$ and $\bar{u}$ are Lipschitz concave functions with respect to Wasserstein distance $W_{p}$ of order $p$ and satisfy positive homogeneity.

Proof: We only need to prove that expressions (13) and (14) hold, and the rest conclusions can be obtained from theorem 1.1.

Since the strategies of both sides of the game are independent of $L$, so (11) can be equivalent to:

$$
\underline{u}(\mu):=\sup _{\sigma \in \Delta(I) \tau \in \Delta(J)} \inf _{\pi(\mu, \sigma, \tau)}\left[\left\langle E[L], T\left(i_{q}, j_{q}\right)\right\rangle\right] 。
$$

In Formula (4), taking $n=1, L=E[L]=E(\mu)$, we can see that Formula (13) holds, and Formula (14) can be similarly verified.

\section{3. $\operatorname{Cav}(u)$ theorem}

In [9], the $\operatorname{Cav}(\mathrm{u})$ theorem for $\mu \in \Delta(P) \subset \Delta^{1}\left(\boldsymbol{R}^{d}\right)$ is proved, where $P$ is the convex compact subset of $\boldsymbol{R}^{d}$ convex compact subset. The following theorem summarizes Theorem 1.1 and Proposition 2.4 in [9].

Theorem 2.1 For all $\mu \in \Delta(P)$, there is:

$$
\lim _{n \rightarrow \infty} \frac{1}{n} \underline{V}_{n}(\mu)=\operatorname{Cav}(\underline{u})(\mu)
$$




$$
\lim _{n \rightarrow \infty} \frac{1}{n} \bar{V}_{n}(\mu)=\operatorname{Cav}(\bar{u})(\mu)
$$

Where $\operatorname{Cav}(\cdot)$ is the concave operator on $\Delta(P)$, namely $\operatorname{Cav}(u)=\inf \{f \in \operatorname{USCC}: f \geq u\}$, and USCC represents the set of upper semi-continuous concave functions.

In order to extend the $\operatorname{Cav}(\mathrm{u})$ theorem to $\Delta^{p}\left(\boldsymbol{R}^{d}\right)$, a lemma is first given.

Lemma 2.2 For any $\mu \in \Delta^{p}\left(\boldsymbol{R}^{d}\right), \varepsilon>0$, there exists a convex compact subset $P \in \boldsymbol{R}^{d}$ and a probability measure $\bar{\mu}$ on $P$, such that:

$$
W_{p}(\mu, \bar{\mu}) \leq \varepsilon
$$

Proof: For any $\varepsilon>0$, the existence of $N \geq 1$ makes:

$$
\int_{\{|x|>N\}}|x|^{p} d \mu(x)<\frac{\varepsilon^{p}}{4^{p}}
$$

Let $P=\left\{x \in \boldsymbol{R}^{d}:|x| \leq N+1\right\}, \bar{P}=\left\{x \in \boldsymbol{R}^{d}:|x| \leq N\right\}$. Obviously, $P$ is convex compact sets.

Take $\bar{\mu}$ defined on $P$ and satisfy $\bar{\mu}=\mu$ on $\bar{P}$. Therefore:

$$
\bar{\mu}(\{N<|x| \leq N+1\})=\mu(\{|x|>N\})<\frac{\varepsilon^{p}}{4^{p} N^{p}}
$$

According to the theorem 7.10 in Villani, we can get:

$$
\begin{aligned}
W_{p}(\mu, \bar{\mu})^{p} & \leq 2^{p-1} \int_{\boldsymbol{R}^{d}}|x|^{p} d|\mu-\bar{\mu}|(x) \\
& \leq 2^{p-1} \int_{\{|x|>N\}}|x|^{p} d \mu(x)+\int_{\{N<|x| \leq N+1\}}|x|^{p} d \bar{\mu}(x) \\
& \leq 2^{p-1}\left(\frac{\varepsilon^{p}}{4^{p}}+\frac{(N+1)^{p} \varepsilon^{p}}{4^{p} N^{p}}\right) \\
& \leq 2^{p-1}\left(\frac{\varepsilon^{p}}{4^{p}}+\frac{\varepsilon^{p}}{2^{p}}\right)<\varepsilon^{p} 。
\end{aligned}
$$

The $\operatorname{Cav}(\mathrm{u})$ theorem with $\mu \in \Delta^{p}\left(\boldsymbol{R}^{d}\right)$ is given below.

Theorem 2.3 For all $\forall \mu \in \Delta^{p}\left(\mathbf{R}^{d}\right)$, there are:

$$
\lim _{n \rightarrow \infty} \frac{1}{n} \underline{V}_{n}(\mu)=\operatorname{Cav}(\underline{u})(\mu)
$$




$$
\lim _{n \rightarrow \infty} \frac{1}{n} \bar{V}_{n}(\mu)=\operatorname{Cav}(\bar{u})(\mu)
$$

Proof: Here, only the proof of Equation (22) is given. In fact, since $\frac{1}{n} \underline{V}_{n}(\mu) \geq \underline{u}(\mu)$ and $\underline{V}_{n}$ are concave functions on $\Delta^{p}\left(\boldsymbol{R}^{d}\right)$, we can easily find that for every $\mu \in \Delta^{p}\left(\boldsymbol{R}^{d}\right)$,

$$
\operatorname{Cav}(\underline{u})(\mu) \leq \frac{1}{n} \underline{V}_{n}(\mu)
$$

Because $\underline{V}_{n}$ and $\underline{u}$ are Lipschitz continuous, $\frac{1}{n} \underline{V}_{n}$ is weakly continuous on $\Delta^{p}\left(\boldsymbol{R}^{d}\right)$ and the $\operatorname{Cav}(\underline{u})$ is weakly upper semicontinuous on $\Delta^{p}\left(\boldsymbol{R}^{d}\right)$. For every $\varepsilon>0$, according to the Lemma 2.2, we can take $\bar{\mu} \in \Delta^{p}\left(\boldsymbol{R}^{d}\right)$, where $P$ is a convex compact set in $\boldsymbol{R}^{d}$ such that:

$$
\begin{gathered}
\left|\frac{1}{n} \underline{V}_{n}(\mu)-\frac{1}{n} \underline{V}_{n}(\bar{\mu})\right| \leq \varepsilon, \forall n \in N, \\
\operatorname{Cav}(\underline{u})(\bar{\mu}) \leq \operatorname{Cav}(\underline{u})(\mu)+\varepsilon_{0}
\end{gathered}
$$

Thus, according to the Theorem 2.1, we can obtain that:

$$
\begin{aligned}
\underset{n \rightarrow \infty}{\limsup } \frac{1}{n} \underline{-}_{n}(\mu) & \leq \underset{n \rightarrow \infty}{\limsup } \frac{1}{n} \underline{-}_{n}(\bar{\mu})+\limsup _{n \rightarrow \infty}\left|\frac{1}{n} \underline{V}_{n}(\mu)-\underline{V}_{n}(\bar{\mu})\right| \\
& \leq \operatorname{Cav}(\underline{u})(\bar{\mu})+\varepsilon \\
& \leq \operatorname{Cav}(\underline{u})(\mu)+2 \varepsilon
\end{aligned}
$$

Equations (24) and (25) are synthesized, namely, the proof formula (22) is established. Formula (23) can be proved by the same principle.

Corollary 2.4 Given $\mu \in \Delta^{p}\left(\boldsymbol{R}^{d}\right)$, if $\bar{u}(\mu)=\underline{u}(\mu)$ is satisfied, namely, the value of a single round full information game exists, then:

$$
\lim _{n \rightarrow \infty} \frac{1}{n} \underline{V}_{n}(\mu)=\lim _{n \rightarrow \infty} \frac{1}{n} \bar{V}_{n}(\mu)=\operatorname{Cav}(\bar{u})(\mu)
$$




\section{Conclusion}

In this paper, we considered the repeated game model $\Gamma_{n}(\mu)$ with one-sided incomplete information when the state set and strategy set are infinite sets, and the Cav(u) theorem was given when $\mu \in \Delta^{p}\left(\boldsymbol{R}^{d}\right)$ holds. The properties of Wasserstein distance used in the proof provides an approximation method for dealing with the probabilistic measure problem of the whole space.

\section{Funding Statement}

This paper was funded by the National Social Science Foundation of China (No.18CJY008).

\section{References}

[1] R. J. Aumann and M. Maschler. Repeated Games with Incomplete Information. Cambridge: MIT Press. 1953.

[2] De Meyer B, Lehrer E, Rosenberg D. Evaluating information in zero-sum games with incomplete information on both sides. Math. Oper. Res. 2010, vol. 35, no. 4, 851.

[3] Renault, Jérôme. The Value of Markov Chain Games with Lack of Information on One Side. Mathematics of Operations Research. 2006, vol. 31, no. 3, 490-512.

[4] Gensbittel Fabien, Renault Jérôme. The Value of Markov Chain Games with Incomplete Information on Both Sides. Mathematics of Operations Research. 2015, vol. 40, no. 4, $820-841$.

[5] Neyman, A. Existence of optimal strategies in Markov games with incomplete information. Int J Game Theory. 2008, 581-596.

[6] De Meyer B, Rosenberg D. 'Cav u' and the Dual Game. Mathematics of Operations Research. 1999, vol. 24, 619-626.

[7] De Meyer, B. Price dynamics on a stock market with asymmetric information. Games and Economic Behavior. 2010, vol. 69, no. 1, 42-71. 
[8] Fabien Gensbittel. Extensions of the Cav(u) theorem for repeated games incomplete information on one side. Mathematics of Operations Research. 2015, vol. 40, no. 1, 80-104.

[9] GENSBITTEL. F. Analyse asymptotique de jeux répétés à information incomplete. Paris: University of Paris. 2010.

[10] Laraki R. Repeated games with lack of information on one side: The dual differential approach. Math. Oper. Res. 2002 vol. 27, no. 2, 419-440.

[11] Pedro C. Alvarez-Esteban, Eustasio del Barrio, Juan A. Cuesta-Albertos et al. Assessing when a sample is mostly normal. Computational Statistics \& Data Analysis. 2010, vol. 54, no. $12,2914-2925$.

[12] Juan F. Escobar, Gaston Llanes. Cooperation dynamics in repeated games of adverse selection. Journal of Economic Theory. 2018, vol. 176, 408-443.

[13] Ashkenazi-Golan, G, Rainer, C, Solan, E. Solving two-state Markov games with incomplete information on one side. Games and Economic Behavior. 2020, vol. 122, 83-104.

[14] Ueda, Masahiko. Tit-for-Tat Strategy as a Deformed Zero-Determinant Strategy in Repeated Games. Journal of the Physical Society of Japan. 2021, vol. 90, no. 2.

[15] SORIN. S. A first course on zero-sum repeated games. Paris: Springer. 2002.

[16] VILLANI. C. Topics in optimal transportation. Providence: American Mathematical Society. 2003.

[17] V. M. Panaretos and Y. Zemel. Statistical Aspects of Wasserstein Distances. Annual Review of Statistics and Its Application. 2018, vol. 6, no. 1, 405-431. 